\title{
Expression and clinical significance of autophagic protein LC3B and EMT markers in gastric cancer
}

This article was published in the following Dove Press journal:

Cancer Management and Research

\author{
Juan-Yi Wang' \\ Tao $\mathrm{Wu}^{2}$ \\ $\mathrm{Wu} \mathrm{Ma}{ }^{1}$ \\ Shuo Li' \\ Wen-Jiang Jing' \\ Jun $\mathrm{Ma}^{\mathrm{I}}$ \\ Dong-Mei Chen' \\ Xiao-Jie Guo' \\ Ke-Jun $\mathrm{Nan}^{3}$
}

'Department of Oncology, 320।

Affiliated Hospital of Medical

College of Xi'an Jiaotong University,

Hanzhong, Shaanxi, People's Republic

of China; ${ }^{2}$ Department of Gynecologic

Cancer, Shaanxi Province Tumor

Hospital, Xi'an, Shaanxi, People's

Republic of China; ${ }^{3}$ Department of

Medical Oncology, The First Affiliated

Hospital, College of Medicine of Xi'an

Jiaotong University, Xi'an, Shaanxi,

People's Republic of China
Correspondence: Ke-Jun Nan

Department of Medical Oncology, The

First Affiliated Hospital, College of

Medicine of Xi'an Jiaotong University,

No. 277 Yanta West Road, Xi'an, Shaanxi

7I006I, People's Republic of China

Tel +862985324086

Fax +862985324086

Email nankj@I63.com
Background and aim: Gastric cancer (GC) is a fatal malignancy with high rate of recurrence and metastasis. Here, we investigated the correlations between the expression of autophagic protein LC3B and 2 epithelial-mesenchymal transition-related proteins (E-cadherin and Vimentin) and the clinicopathological factors and prognosis of patients with GC.

Materials and methods: The expression of LC3B, E-cadherin, and Vimentin in GC samples (110 cases) and paracarcinoma tissues (40 cases) was analyzed using the Oncomine databases and further detected by immunohistochemistry. The correlations among these markers expression and clinicopathological features in GC were analyzed. The patients were followed for survival analysis

Results: Compared to the nontumor tissues, the expression of LC3B and Vimentin proteins were significantly elevated in GC tissues, but the E-cadherin expression was decreased (all $p<0.05$ ). Interestingly, LCB expression was positively correlated with Vimentin $(r=0.320, p=0.001)$ and negatively associated with E-cadherin expression $(r=-0.484, p<0.001)$ in GC. The expression of these markers was closely related to tumor differentiation, T classification, TNM stage, and lymph node metastasis (all $p<0.05$ ). Furthermore, survival analyses and screening Kaplan-Meier plotter database revealed that GC patients with high LC3B and Vimentin expression levels had a poorer clinical outcome than those with low expression. Conversely, high E-cadherin expression was linked with favorable overall survival (all $p<0.05$, log-rank test). Multivariate survival analysis demonstrated that LC3B, E-cadherin, and Vimentin expression were independent prognostic factors of GC patients.

Conclusion: LC3B, E-cadherin, and Vimentin may play an important role in the tumorigenesis of GC, and these marker expressions may serve as additional prognostic indicators for overall survival of patients. The interactions of autophagy and epithelial-mesenchymal transition in GC merits further investigation.

Keywords: autophagy, epithelial-mesenchymal transition, gastric cancer, prognosis, KaplanMeier plotter database

\section{Introduction}

Gastric cancer (GC) represents one of the most aggressive malignant diseases with a high mortality rate globally. It still remains the leading causes of cancer-related death in Asia, in spite of its declining incidence worldwide. ${ }^{1}$ Surgery is the cornerstone of therapy in early stage GC patients. However, most patients are in advanced or distant metastatic disease at diagnosis, and thus surgery is limited, resulting in a 5-year overall survival (OS) rate as low as 29\%. ${ }^{2}$ Clinicopathological parameters (TNM staging, grade of differentiation, and histological type) are used to predict patients with prognosis 
and help guide therapeutic decisions, which, however, have limitation because of heterogeneity of patients. ${ }^{3}$ Therefore, it is of great clinical significance to identify new additional prognostic markers in order to improve individual patient care.

Autophagy is a self-degradative process for the degradation and recycling of cytoplasmic components such as proteins and damaged organelles. The process is tightly regulated and highly conserved to ensure homeostasis. ${ }^{4}$ Autophagy has complex and context-dependent roles in cancer, which depends on tumor stage, genetic context, and tumor microenvironment. ${ }^{5}$ The modulation of autophagy has been considered as a therapeutic approach for targeting cancer. In addition, studies reported that autophagy-related markers were associated with prognosis of cancer. ${ }^{6,7} \mathrm{LC} 3 \mathrm{~B}$ is widely used as autophagy marker. Immunohistochemical LC3B staining is considered to be indicative of basal autophagy inpatient tissue. ${ }^{89}$ LC3B protein has been proposed as potential prognostic biomarker for various tumors, including melanoma, ${ }^{10}$ esophageal adenocarcinomas, ${ }^{8}$ and breast cancer. ${ }^{6}$ However, there is sparse data on the prognostic role of LC3B in patients with GC.

The process whereby epithelial cells take on characteristics of mesenchymal cells is termed epithelial to mesenchymal transition (EMT). ${ }^{11}$ The EMT process involves downregulation of the expression of E-cadherin, which is a well-established epithelial marker, and upregulation of Vimentin. ${ }^{12}$ Studies have reported that EMT has a critical role for contributing to invasive and metastatic potential of cancer. Recent researches in ovarian cancer, nonsmall-cell lung cancer, colorectal cancer, and GC ${ }^{13-15}$ have shown that EMT-related markers were implicated as critical predictors for prognosis. Previously, EMT- and autophagy-related markers were separately reported regarding their prognostic effects on GC. ${ }^{15,16}$ Recent observations indicate that the interaction between autophagy and EMT exists in cancer. ${ }^{17}$ However, the complex relationship about the 2 processes are not definitive yet in GC. In addition, their mutual associations in a clinical setting remain elusive.

Therefore, we conducted the present study to determine the correlations between the expression of autophagic protein LC3B and 2 EMT-related proteins E-cadherin and Vimentin, and the clinicopathological factors and prognosis of GC.

\section{Materials and methods Patients}

GC samples (110 cases) and paracarcinoma tissues (40 cases), obtained from 3201 Affiliated Hospital of Medical College of Xi'an Jiaotong University from 2010 to
2014, were used in the current study. All the patients with GC underwent a radical resection from the same surgical team and were histologically confirmed to have gastric adenocarcinoma. The patients did not receive preoperative chemotherapy and/or radiotherapy. Two independent gastroenterology pathologists assessed the tumor-related clinicopathologic factors by pathological examination. The clinicopathological parameters, including age, gender, tumor differentiation, stage, and lymph node metastasis, were retrospectively collected. These variables are listed in Table 1. Follow-up information was obtained by registered telephone, mail, or outpatient service. OS was calculated as the time from the date of surgery to death from any cause or the last follow-up date. Survival data were collected until October 2017 or until the date of patient death.

\section{Immunohistochemistry and evaluation of immunostaining}

Tissue samples were formalin fixed and paraffin embedded. streptavidin-perosidase (SP) immunohistochemistry staining was performed in strict accordance with the instructions of the kit (SP Staining Kits; BIOSS Biotechnology Co., Ltd., Beijing, People's Republic of China). Antigen retrieval was done by a combination of heat and pressure in sodium citrate buffer (pH 6.0). Sections were incubated with rabbit polyclonal primary antibody anti-LC3B (dilution, 1:500; cat. no. bs-4843R; BIOSS), E-cadherin (dilution, 1:500; cat. no. bs-1519R; BIOSS), and Vimentin (dilution, 1:200; cat. no. bs-0756R; BIOSS) overnight at $4^{\circ} \mathrm{C}$. After incubation with coordinate secondary antibody, staining was displayed with DBA solution. The nuclei were lightly counterstained with hematoxylin and sections were visualized through a light microscope (Olympus BH-12, Olympus Optical Co., Ltd., Tokyo, Japan) at magnification $400 \times$. The immunoreactivity was evaluated according to the cell staining intensity and percentage of positive areas, as described previously. ${ }^{18}$ The percentage of staining was semiquantitatively scored as follows: $0 \%$ number of positive stained cell scored $0,<10 \%$ scored $1,10 \%-50 \%$ scored 2 , and $>50 \%$ scored 3 . The intensity of staining was as follows: no staining (colorless) scored 0 , weak staining (pale yellow) scored 1, moderate staining (yellow) scored 2, and strong staining (brown) scored 3. The staining index was generated by multiplying the intensity score and the score of the percentage of positive cells. The final scores of $<4$ were noted as low expression, and the remaining were designated as high expression. Two pathologists who were blinded to patient clinicopathological data independently assessed the immunostaining results. 
Table I Relationship between LC3B, E-cadherin, and Vimentin expression and clinicopathological characteristics in GC

\begin{tabular}{|c|c|c|c|c|c|c|c|c|c|c|}
\hline \multirow[t]{2}{*}{ Variables } & \multirow[t]{2}{*}{$\mathbf{N}$} & \multicolumn{2}{|c|}{ LC3 expression } & \multirow[t]{2}{*}{$p$-value } & \multicolumn{2}{|c|}{ E-cadherin expression } & \multirow[t]{2}{*}{$p$-value } & \multicolumn{2}{|c|}{ Vimentin expression } & \multirow[t]{2}{*}{$p$-value } \\
\hline & & High (\%) & Low (\%) & & High (\%) & Low (\%) & & High (\%) & Low (\%) & \\
\hline \multicolumn{11}{|l|}{ Gender } \\
\hline Male & 89 & $56(62.9)$ & $33(37.1)$ & 0.464 & $28(3 \mid .5)$ & $61(68.5)$ & 0.767 & $66(74.2)$ & $23(25.8)$ & 0.123 \\
\hline Female & 21 & $15(7 \mid .4)$ & $6(28.6)$ & & $6(28.6)$ & $15(7 \mid .4)$ & & $12(57.1)$ & $9(42.9)$ & \\
\hline \multicolumn{11}{|l|}{ Age (years) } \\
\hline$\geq 60$ & 48 & $33(68.8)$ & $15(3 \mid .2)$ & 0.417 & $17(35.4)$ & $31(64.6)$ & 0.368 & $32(66.6)$ & $16(33.3)$ & 0.628 \\
\hline$<60$ & 62 & $38(61.3)$ & $24(38.7)$ & & $17(27.4)$ & $45(72.6)$ & & $44(72.6)$ & $18(27.4)$ & \\
\hline \multicolumn{11}{|l|}{ T classification } \\
\hline $\mathrm{TI} / \mathrm{T} 2$ & 17 & $6(35.3)$ & II (64.7) & 0.006 & $9(52.9)$ & $8(47.1)$ & 0.033 & $8(47.1)$ & $9(52.9)$ & 0.039 \\
\hline $\mathrm{T} 3 / \mathrm{T} 4$ & 93 & $65(69.9)$ & $28(30.1)$ & & $25(26.9)$ & $68(73.1)$ & & $70(75.3)$ & $23(24.7)$ & \\
\hline \multicolumn{11}{|l|}{ Nodal involvement } \\
\hline Negative & 36 & $18(50.0)$ & $18(50.0)$ & 0.026 & $16(44.4)$ & $20(55.6)$ & 0.032 & $20(55.6)$ & $16(44.4)$ & 0.013 \\
\hline Positive & 74 & $53(71.6)$ & $21(28.4)$ & & $18(24.3)$ & $56(73.7)$ & & $58(78.4)$ & $16(21.6)$ & \\
\hline \multicolumn{11}{|l|}{ TNM stage } \\
\hline I+II & 44 & $20(45.5)$ & $24(54.5)$ & 0.001 & $20(45.5)$ & $24(54.5)$ & 0.007 & $18(40.9)$ & $26(59.1)$ & 0.000 \\
\hline III+IV & 66 & $51(77.3)$ & I5 (22.7) & & $14(2 \mid .2)$ & $52(78.8)$ & & $60(90.0)$ & $6(9.1)$ & \\
\hline \multicolumn{11}{|l|}{ Tumor size } \\
\hline$\geq 5 \mathrm{~cm}$ & 64 & $40(62.5)$ & $24(37.5)$ & 0.597 & $18(28.1)$ & 46 (7I.9) & 0.456 & $42(65.6)$ & $22(34.4)$ & 0.150 \\
\hline$<5 \mathrm{~cm}$ & 46 & $31(67.4)$ & $15(32.6)$ & & $16(34.8)$ & $30(65.2)$ & & $36(78.3)$ & $10(21.7)$ & \\
\hline \multicolumn{11}{|l|}{ Differentiation } \\
\hline Low & 50 & $39(78.0)$ & II (22.0) & 0.007 & $10(20.0)$ & $40(80.0)$ & 0.024 & $31(68.0)$ & $19(32.0)$ & 0.060 \\
\hline High/moderate & 60 & $32(53.3)$ & $28(46.7)$ & & $24(40.0)$ & $36(60.0)$ & & 47 (78.3) & $13(2 \mid .7)$ & \\
\hline
\end{tabular}

Note: Bold values have statistical significance.

Abbreviation: GC, gastric cancer.

\section{Bioinformatics analysis}

To determine the expression pattern of the LC3B, E-cadherin, and Vimentin genes in GC, a search of the publicly available Oncomine database (http://www.oncomine.com, Compendia Bioscience, Inc, Ann Arbor, MI, USA) was initially conducted. Briefly, the 3 genes were queried in the database, and the results were displayed by using Box chart. The data type was restricted to mRNA. GC versus normal tissues were compared in the differential analysis. We set up the $p$-value as 0.05 and gene rank to top $10 \%$. The prognostic value of the LC3B, E-cadherin, and Vimentin mRNA expression in GC was also analyzed using the Kaplan-Meier Plotter (http://kmplot.com/analysis/). Currently, the database encompasses 54,675 genes, and their effect on survival is assessed using 10,461 cancer samples. ${ }^{19}$ The database was used as previously described..$^{20}$ Briefly, the genes were entered into the database of GC to obtain Kaplan-Meier survival plots. The Multivariate Cox regression model was used and HR, 95\% CI, and log-rank $p$-value were calculated and displayed.

\section{Statistical analysis}

Categorical variables were compared using the chi-squared test or Fisher's exact test. Spearmen's rank correlation analysis was applied to analyze the relationship between
LC3B- and EMT-related markers. OS curves were estimated using the Kaplan-Meier method and evaluated using the log-rank test. Multivariate analysis on prognostic variables was performed using the Cox proportional hazard regression model with stepwise forward selection. Statistical analyses were performed using SPSS 23.0 software (IBM Corporation, Armonk, NY, USA). Statistical significance was defined as $p<0.05$.

\section{Ethics statements}

The Institutional Review Board of 3201 Affiliated Hospital of Medical College of Xi'an Jiaotong University (Han-zhong, People's Republic of China) approved this research. Written informed consent was obtained from all participants.

\section{Results \\ Expression of LC3B, E-cadherin, and Vimentin in GC and adjacent normal tissues}

First, we evaluated transcription levels of LC3B, E-cadherin, and Vimentin in $\mathrm{GC}$ and normal gastric mucosa using the Oncomine database. In D'Errico et al's ${ }^{21}$ and Chen et al's ${ }^{22}$ data set, the results revealed that LC3B and Vimentin mRNA expression was higher in intestinal, diffuse, and mixed gastric adenocarcinoma compared with gastric mucosa 
(Figure $1 \mathrm{~A}-\mathrm{C}$ and $1 \mathrm{G}-\mathrm{I}$, all $p<0.05$ ). In Cho's data set, ${ }^{23}$ E-cadherin mRNA expression in the gastric adenocarcinoma tissue was markedly lower than in normal tissue (Figure 1D-F, all $p<0.05$ ). Then, as shown in Figure 2, we detected LC3B, E-cadherin, and Vimentin protein expression in GC and adjacent tissues using immunohistochemistry (IHC). Consistent with mRNA expression, as shown in Table 2, the high expression rates of LC3B and Vimentin in GC tissues were $64.5 \%$ (71/110) and $70.9 \%(78 / 110)$, respectively, which were higher than those in adjacent tissues (LC3B, 20\% [8/40], $p<0.001$; Vimentin, 30\% [12/40], $p<0.001)$. The most adjacent normal tissues $(77.5 \%$, $31 / 40$ ) showed higher levels of E-cadherin protein compared with the corresponding GC tissues $(30.9 \%, 34 / 110, p<0.001)$.

\section{Correlations between LC3B, E-cadherin, and Vimentin expression and clinicopathological factors in GC}

As listed in Table 1, the LC3B expression was associated with the $\mathrm{T}$ classification $(p=0.006)$, lymph node metastasis ( $p=0.026)$, TNM stage $(p=0.001)$, and the degree of differentiation $(p=0.007)$. Significant associations between
E-cadherin expression and T classification ( $p=0.033)$, nodal involvement $(p=0.032)$, TNM stage $(p=0.007)$, and histological differentiation $(p=0.024)$ were identified. Vimentin expression was closely related to $\mathrm{T}$ classification $(p=0.039)$, nodal involvement $(p=0.013)$, and TNM stage $(p=0.000)$. LC3B, E-cadherin, and Vimentin expression were unrelated to age, gender, and tumor size (all $p>0.05$ ).

\section{LC3B expression correlated with E-cadherin and Vimentin in GC}

We next evaluated the correlations between LC3B and E-cadherin and Vimentin expression in GC tissues. As shown in Table 3, the Spearman rank correlation analysis indicated that the expression of LC3B was negatively correlated with E-cadherin expression $(r=-0.484, p<0.001)$ and was positively associated with Vimentin expression $(r=0.320, p=0.001)$.

\section{Survival analysis}

We next examined the prognostic value of LC3B, E-cadherin, and Vimentin mRNA expression in GC using the KaplanMeier plotter database (http://kmplot.com/analysis/). The
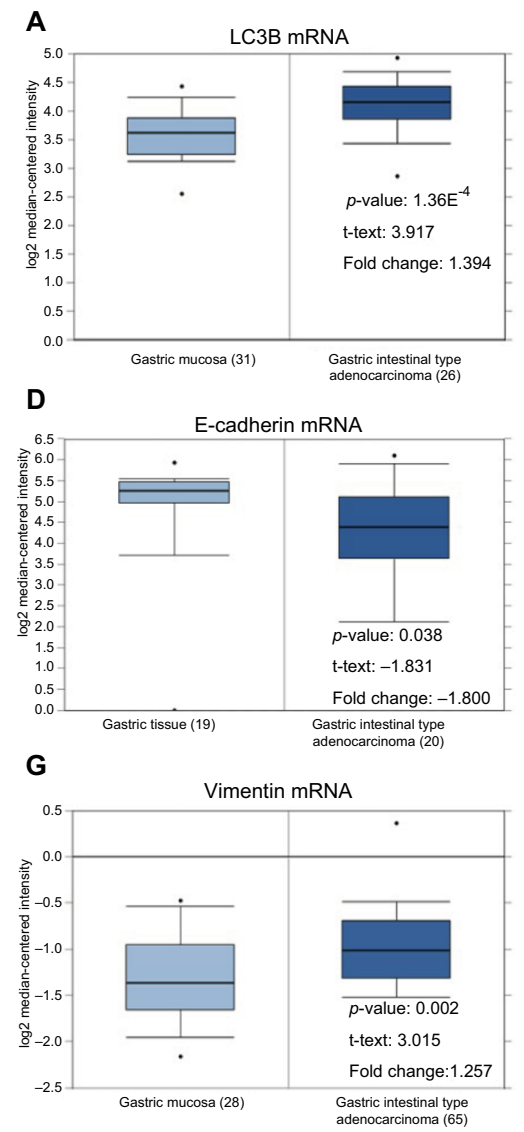

B

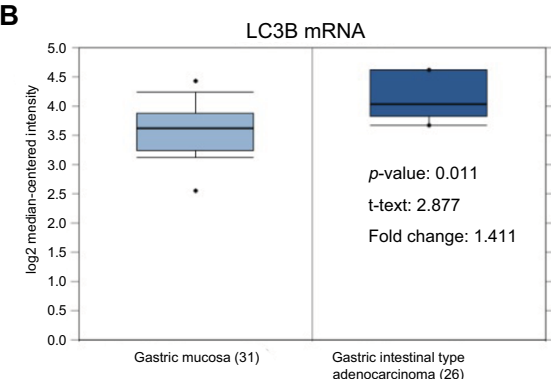

E

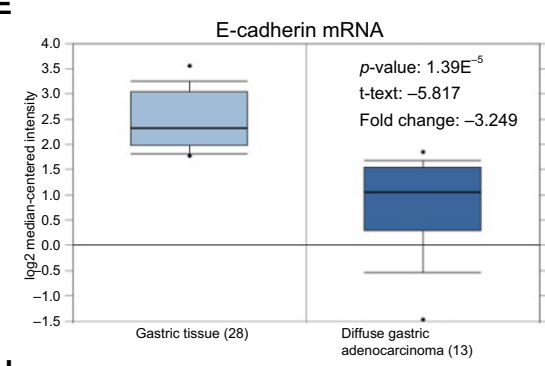

H

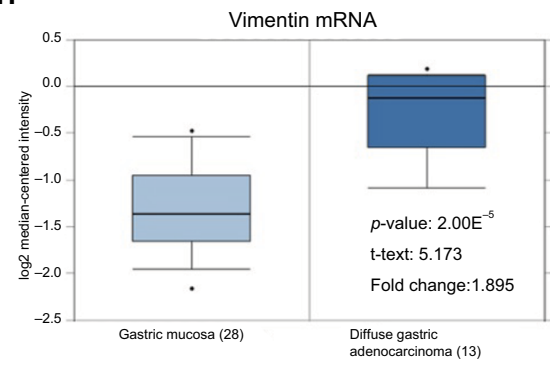

C

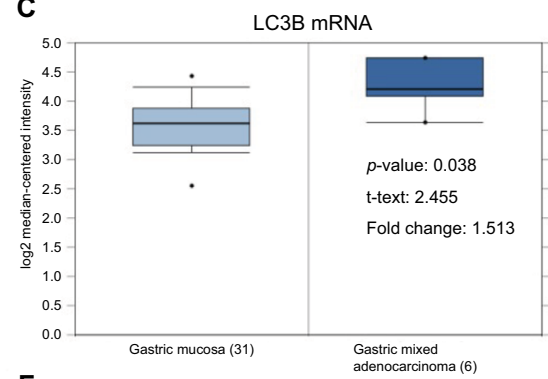

$\mathbf{F}$

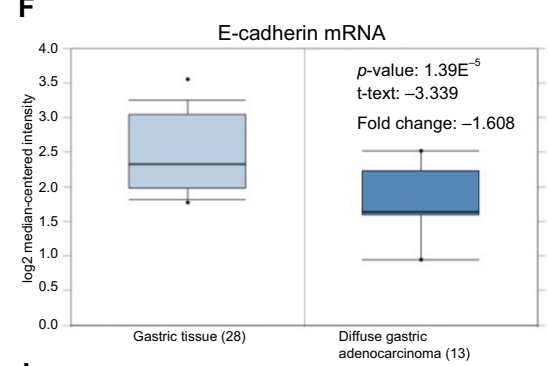

I

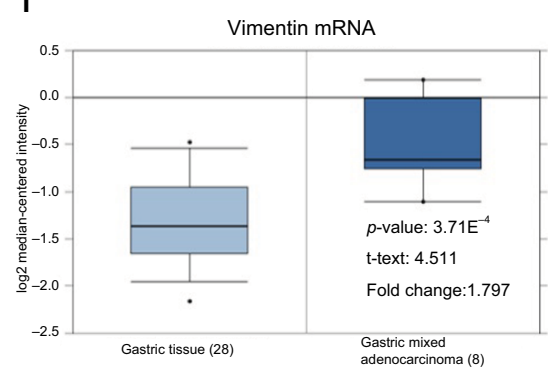

Figure I Box and whiskers plots of Oncomine data on LC3B (A-C), E-cadherin (D-F), and Vimentin (G-I) mRNA levels in GC and normal tissues. Abbreviation: GC, gastric cancer. 


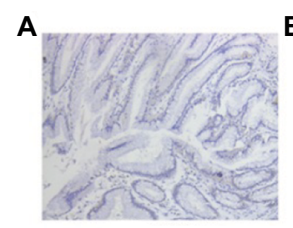

F
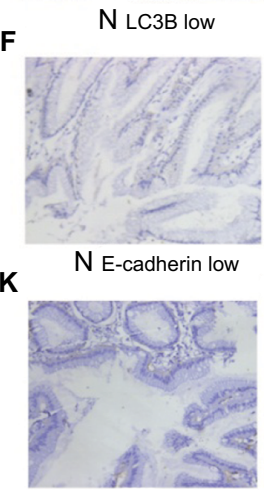

$\mathrm{N}$ Vimentin low
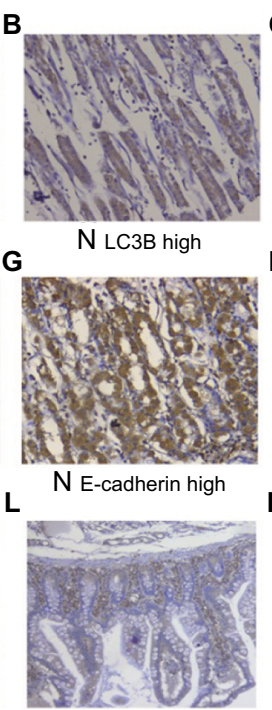

$\mathrm{N}$ Vimentin high

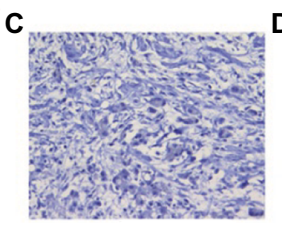

H

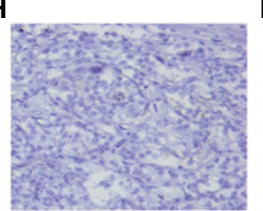

T E-cadherin low

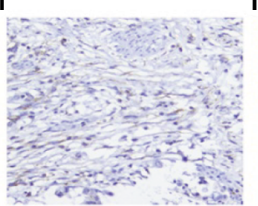

T Vimentin low

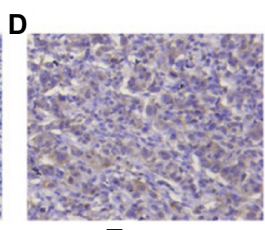

T LC3B high

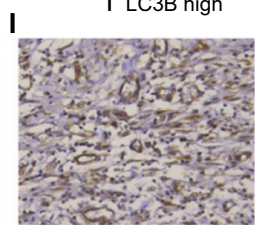

T E-cadherin high

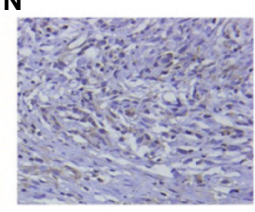

T Vimentin high
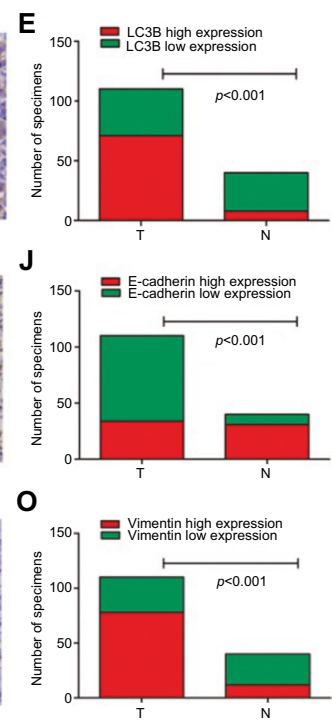

Figure 2 Representative examples of LC3B, E-cadherin, and Vimentin expression (200x).

Notes: Low LC3B expression in paired nontumor (A) and GC tissues (C), high LC3B expression in adjacent normal (B) and GC tissues (D), comparisons of LC3B expression in adjacent normal and GC tissues (E), low E-cadherin expression in adjacent normal $(\mathbf{F})$ and $G C$ tissues $(\mathbf{H})$, high E-cadherin expression in adjacent normal $(\mathbf{G})$ and GC tissues (I), comparisons of E-cadherin expression in adjacent normal and GC tissues (J), low Vimentin expression in adjacent normal (K) and GC tissues (M), high Vimentin expression in adjacent normal (L) and GC tissues (N), comparisons of Vimentin expression in adjacent normal and GC tissues (O).

Abbreviations: GC, gastric cancer; N, adjacent normal tissue; T, GC tissue.

Table 2 Expressions of LC3B, E-cadherin, and Vimentin in GC tissue and $\mathrm{N}$ tissue

\begin{tabular}{|c|c|c|c|c|c|c|c|c|c|c|}
\hline \multirow[t]{2}{*}{ Group } & \multirow[t]{2}{*}{ Simples } & \multicolumn{2}{|c|}{ LC3B expression } & \multirow[t]{2}{*}{$p$-value } & \multicolumn{2}{|c|}{ E-cadherin expression } & \multirow[t]{2}{*}{$p$-value } & \multicolumn{2}{|c|}{ Vimentin expression } & \multirow[t]{2}{*}{$p$-value } \\
\hline & & High (\%) & Low (\%) & & High (\% & Low (\%) & & High (\%) & Low (\%) & \\
\hline GC & 110 & $71(64.5)$ & $39(35.5)$ & 0.000 & $34(30.9)$ & $76(69.1)$ & 0.000 & $78(70.9)$ & $32(29.1)$ & 0.000 \\
\hline $\mathrm{N}$ & 40 & $8(20)$ & $32(80)$ & & 31 (77.5) & $9(22.5)$ & & $12(30.0)$ & $28(70.0)$ & \\
\hline
\end{tabular}

Abbreviations: GC, gastric cancer; $\mathrm{N}$, adjacent normal tissue.

Table 3 Correlations between LC3B with E-cadherin and Vimentin in GC

\begin{tabular}{|c|c|c|c|c|c|}
\hline \multirow[t]{2}{*}{ EMT markers } & \multirow[t]{2}{*}{$\mathbf{N}$} & \multicolumn{2}{|c|}{ LC3B expression } & \multirow[t]{2}{*}{$r$} & \multirow[t]{2}{*}{$p$-value } \\
\hline & & High (\%) & Low (\%) & & \\
\hline E-cadherin expression & & & & -0.484 & $<0.001$ \\
\hline High & 34 & 10 & 24 & & \\
\hline Low & 76 & 61 & 15 & & \\
\hline Vimentin expression & & & & 0.320 & 0.001 \\
\hline High & 78 & 58 & 20 & & \\
\hline Low & 32 & 13 & 19 & & \\
\hline
\end{tabular}

Abbreviations: EMT, epithelial-mesenchymal transition; GC, gastric cancer.

following Affymetrix IDs are valid: 208786_s_at (LC3B), 201131_s_at (E-cadherin), and 201426_s_at (Vimentin). Survival analysis revealed that high expression of LC3B and Vimentin predicted worse OS in GC patients $(\mathrm{N}=876$, $\mathrm{HR}=1.23$ [95\% CI: 1.04-1.46], $p=0.016$, Figure $3 \mathrm{~A}$; $\mathrm{HR}=1.29$ [95\% CI: 1.09-1.53], $p=0.003$; Figure 3C). However, high E-cadherin mRNA high expression was found to be correlated to better $\mathrm{OS}(\mathrm{N}=876, \mathrm{HR}=0.74[95 \% \mathrm{CI}$ :
$0.62-0.87], p<0.001$, Figure 3B). In our cohort, overall, 91 patients died and 19 survived after a median follow-up of 36 months (range, 8-71 months). Consistent with the aforementioned Kaplan-Meier analysis, patients with high LC3B and Vimentin expression exhibited shorter survival than those with low expression (all $p<0.05$, Figure 3D and F). High E-cadherin expression was associated with favorable prognosis in GC patients $(p<0.05$, Figure $3 \mathrm{E})$. Multivariate Cox analysis identified tumor differentiation, TNM stage, $\mathrm{T}$ classification, and lymph node metastasis as significant prognostic factors for OS. In particular, LC3B, E-cadherin, and Vimentin expression were also independently prognostic factors for OS in patients with GC. These analyses are presented in Table 4.

\section{Discussion}

In current study, we evaluated the expression of LC3B- and EMT-related markers (E-cadherin and Vimentin) in GC and the corresponding adjacent normal tissues. In addition, we investigated their relationships with clinicopathological 
A

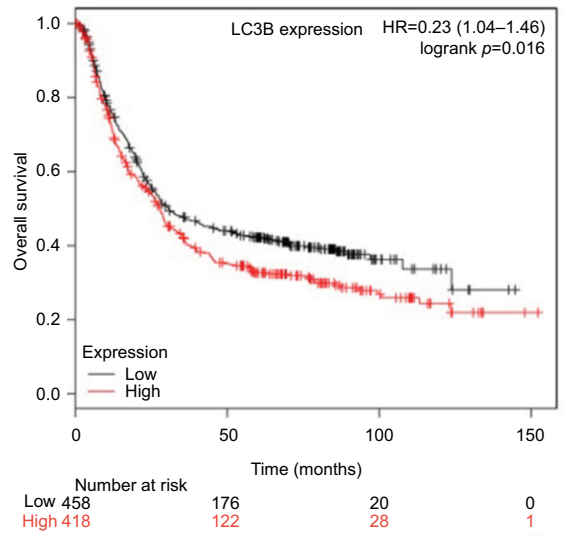

D

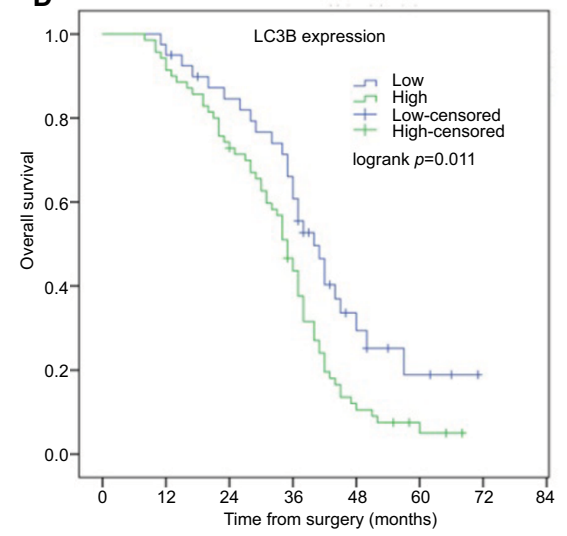

B

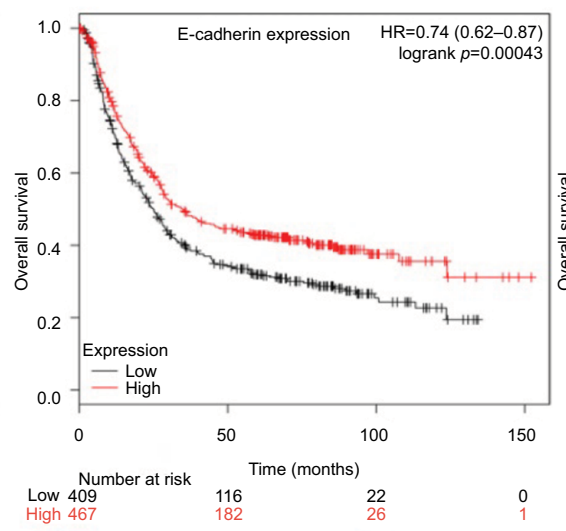

E

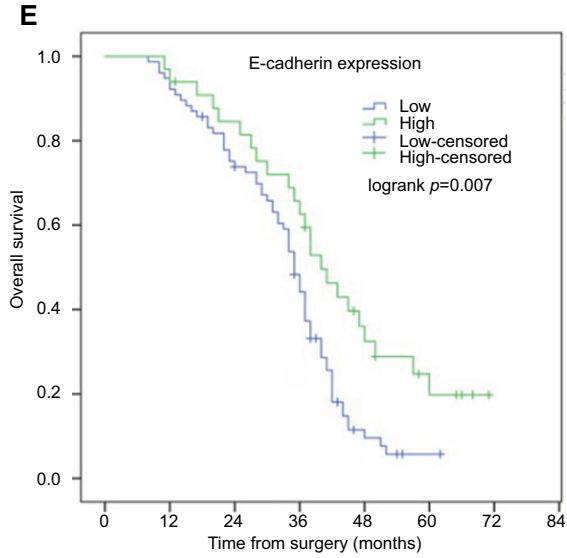

C

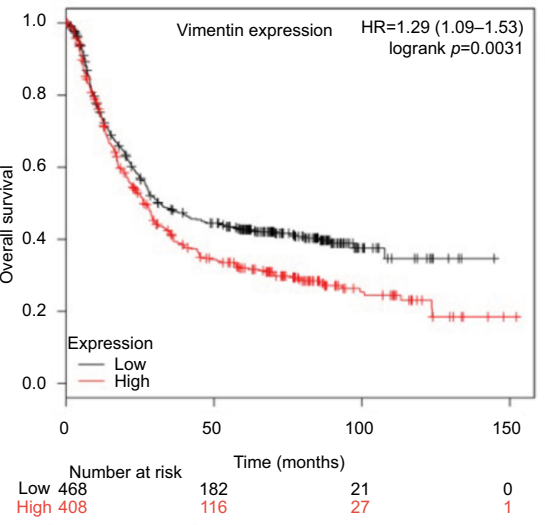

F

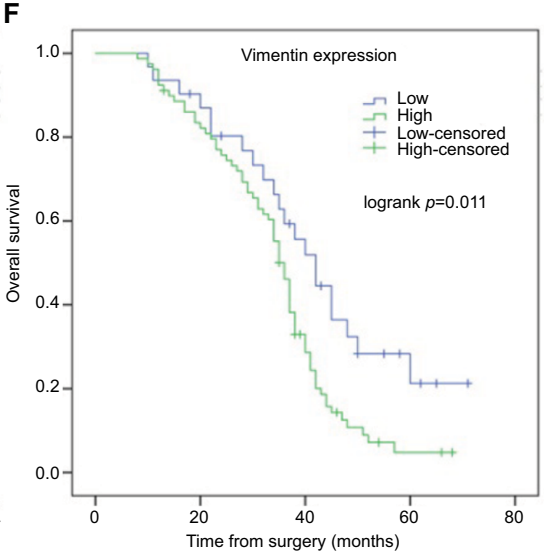

Figure 3 The prognostic value of LC3B, E-cadherin, and Vimentin expression in GC patients.

Notes: OS curves of are plotted for patients with tumors expressing low or high levels LC3B (A), E-cadherin (B), and Vimentin (C) mRNA in Kaplan-Meier plotter database $(\mathrm{N}=876)$. OS curves are plotted for patients with tumors expressing low or high levels LC3B (D), E-cadherin (E), and Vimentin (F) in our retrospective cohort ( $N=1$ I0). $P$-value was calculated by log-rank test and $p<0.05$ was regarded as statistically significant.

Abbreviations: GC, gastric cancer; OS, overall survival.

Table 4 Multivariate analysis of OS for GC patients

\begin{tabular}{|c|c|c|c|}
\hline Variables & HR & $95 \% \mathrm{Cl}$ & $p$-value \\
\hline \multicolumn{4}{|l|}{ Differentiation } \\
\hline Low vs high/moderate & 2.078 & $(1.340-3.222)$ & 0.001 \\
\hline \multicolumn{4}{|l|}{ Nodal involvement } \\
\hline Negative vs positive & 0.387 & $(0.189-0.792)$ & 0.009 \\
\hline \multicolumn{4}{|l|}{ TNM stage } \\
\hline I+II vs III+IV & 0.216 & $(0.103-0.453)$ & 0.000 \\
\hline \multicolumn{4}{|l|}{ T classification } \\
\hline $\mathrm{TI} / \mathrm{T} 2$ vs $\mathrm{T} 2 / \mathrm{T} 3$ & 0.436 & $(0.203-0.936)$ & 0.033 \\
\hline \multicolumn{4}{|l|}{ LC3 expression } \\
\hline High vs low & 1.696 & $(1.078-2.667)$ & 0.022 \\
\hline \multicolumn{4}{|l|}{ Vimentin expression } \\
\hline High vs low & 1.707 & $(1.033-2.819)$ & 0.037 \\
\hline \multicolumn{4}{|l|}{ E-cadherin expression } \\
\hline High vs low & 0.592 & $(0.363-0.967)$ & 0.036 \\
\hline
\end{tabular}

Abbreviations: GC, gastric cancer; OS, overall survival.

factors and clinical outcomes. Our data showed that LC3B, E-cadherin, and Vimentin were universally expressed in GC and corresponding normal tissues. Specifically, LC3B and
Vimentin expression were significantly higher in GC tissues than that in the counterpart normal tissues in both mRNA and protein levels. The expression of E-cadherin in GC tissues was significantly reduced in comparison with normal tissues. LC3B was positively associated with Vimentin and negatively correlated with E-cadherin in GC tissues by Spearman rank correlation analysis. The expression of these markers was closely related to tumor differentiation, $\mathrm{T}$ classification, TNM stage, and nodal involvement. Furthermore, KaplanMeier survival analyses revealed that GC patients with high LC3B and Vimentin expression levels had a worse clinical outcome than those with low expression levels. Conversely, high E-cadherin expression was linked with favorable OS. Multivariate survival analysis demonstrated that LC3B, E-cadherin, and Vimentin expression levels were independent prognostic factors of $\mathrm{GC}$ patients.

GC continues to pose a major challenge in clinical practice with a poor prognosis and limited treatment options. Therefore, it is urgent to identify new additional prognostic 
markers to guide surveillance and improve individual treatment strategies. Mounting studies have indicated that autophagy has an important role in GC development. One study has investigated the prognostic value of autophagy-related proteins in GC and suggested LC3B was not associated with OS. ${ }^{24}$ Yoshioka et $\mathrm{al}^{25}$ has examined LC3 expression in gastrointestinal cancers and found that high protein expression of LC 3 in $58 \%$ of GC (22/38 cases). A recent study by Masuda et $\mathrm{a}^{16}$ evaluated clinicopathological and prognostic significance of LC3 in GC. Consistent with the current study, Masuda et a ${ }^{16}$ report that LC3 was associated with T-stage (depth of tumor invasion) and lymph node metastasis, and suggested that LC3-positive indicated worse outcome in GC than that of patients negative by univariate analysis. Tumor metastasis renders most GC inoperable, resulting in a dismal prognosis. Studies found that autophagy facilitates tumor metastasis through affecting EMT, ${ }^{26}$ tumor angiogenesis, ${ }^{27}$ and inflammatory responses ${ }^{28}$ which may partially explain the LC3B high expression associated with more aggressive behavior of patients with GC. However, some studies ${ }^{29,30}$ revealed that high LC3B expression predicted the poor outcome of triple-negative breast cancer and oral squamous cell carcinoma patients. These findings suggest that autophagy serves multifaceted roles in different types of cancer.

EMT is a process by which epithelial cells dedifferentiate and acquire mesenchymal phenotype. EMT exhibits changes at the molecular level as observed by the decreased expression of E-cadherin and increased expression of Vimentin. Although the role of EMT in cancer is complicated and tissuespecific, ${ }^{31}$ numerous studies that have shown that EMT is a key GC progression driver and facilitates $\mathrm{GC}$ invasion and metastasis. ${ }^{32}$ In line with the previous report, ${ }^{33}$ the present study showed that E-cadherin and Vimentin were prognostic factors of GC. Higher Vimentin and lower E-cadherin protein levels in $\mathrm{GC}$ patients predicted worse OS. Interestingly, the findings of current study suggested that LC3B was linked to EMT-related markers expression in GC. Remarkably, LC3B was positively associated with Vimentin and negatively correlated with E-cadherin in GC tissues. Recent evidence from basic research indicate that autophagy and EMT in cancer are linked in an intricate relationship. ${ }^{34}$ The 2 processes share common molecular mediators and signaling pathways, such as TGF $\beta$, STAT3, and PI3K/AKT/mTOR signaling cascade. Autophagy is a survival-promoting pathway. On the one hand, aberrant EMT activation requires enhanced autophagy to survive during the cancer metastatic spreading under microenvironmental stress. On the other side, autophagy acting as tumor suppression mechanism inhibits the early phases of cancer progression by destabilizing crucial mediators of EMT. ${ }^{34}$ Qiang and $\mathrm{He}^{35}$ reported that autophagy deficiency promotes EMT by SQSTM1-mediated TWIST1 stabilization. Zhao et $\mathrm{a}^{36}$ found that the inhibition of autophagy promotes EMT through increasing ROS/HO-1 signaling pathway in ovarian cancer cells. At present, the precise interactions of autophagy and EMT in GC still remain obscure. Thus, the pivotal molecular mechanism behind the regulation of autophagy and EMT should be further explored.

Several potential limitations should be acknowledged for the current study. First, the numbers of samples are limited, which influences the power of statistical analysis. Second, this was a retrospective study using single-institutional medical information. Certain inherent biases exist. Future larger prospective studies with large samples size may be needed to validate our current data. Finally, the results of our study relied solely on histological examination, and many more in vivo interactions would need to be further researched.

\section{Conclusion}

In summary, LC3B, E-cadherin, and Vimentin may serve as potential prognostic biomarkers for GC; however, much more evidence is needed to prove this. There are correlations between autophagy and EMT in GC based on our study, but their complex interactions require further studies to explore the molecular mechanisms involved. The modulation of autophagy and EMT could be promising targets for the treatment of GC.

\section{Acknowledgment}

The present study was supported by grants from the scientific research project of 3201 Affiliated Hospital of Medical College of Xi'an Jiaotong University (No. 3201ky201629).

\section{Disclosure}

The authors report no conflicts of interest in this work.

\section{References}

1. Van Cutsem E, Sagaert X, Topal B, Haustermans K, Prenen H. Gastric cancer. Lancet. 2016;388(10060):2654-2664.

2. Miller KD, Siegel RL, Lin CC, et al. Cancer treatment and survivorship statistics, 2016. CA Cancer J Clin. 2016;66(4):271-289.

3. Wong SS, Kim KM, Ting JC, et al. Genomic landscape and genetic heterogeneity in gastric adenocarcinoma revealed by whole-genome sequencing. Nature Commun. 2014;5:5477.

4. Levy JMM, Towers CG, Thorburn A. Targeting autophagy in cancer. Nature Rev Cancer. 2017;17(9):528-542.

5. Kimmelman AC. The dynamic nature of autophagy in cancer. Genes Dev. 2011;25(19):1999-2010. 
6. Ladoire S, Penault-Llorca F, Senovilla L, et al. Combined evaluation of LC3B puncta and HMGB1 expression predicts residual risk of relapse after adjuvant chemotherapy in breast cancer. Autophagy. 2015;11(10):1878-1890.

7. Giatromanolaki AN, Charitoudis GS, Bechrakis NE, et al. Autophagy patterns and prognosis in uveal melanomas. Mod Pathol. 2011;24(8): 1036-1045.

8. Adams O, Dislich B, Berezowska S, et al. Prognostic relevance of autophagy markers LC3B and p62 in esophageal adenocarcinomas. Oncotarget. 2016;7(26):39241-39255.

9. Ladoire S, Chaba K, Martins I, et al. Immunohistochemical detection of cytoplasmic LC3 puncta in human cancer specimens. Autophagy. 2012;8(8):1175-1184

10. Tang DYL, Ellis RA, Lovat PE. Prognostic impact of autophagy biomarkers for cutaneous melanoma. Front Oncol. 2016;6:236.

11. Zheng X, Carstens JL, Kim J, et al. Epithelial-to-mesenchymal transition is dispensable for metastasis but induces chemoresistance in pancreatic cancer. Nature. 2015;527(7579):525-530.

12. Okabe H, Mima K, Saito S, et al. Epithelial-mesenchymal transition in gastroenterological cancer. J Cancer Metastasis Treat. 2015;1(3):183-189.

13. Takai M, Terai Y, Kawaguchi H, et al. The EMT (epithelial-mesenchymal-transition)-related protein expression indicates the metastatic status and prognosis in patients with ovarian cancer. JOvarian Res. 2014;7:76.

14. Yang Z, Wang H, Xia L, et al. Overexpression of PAK1 correlates with aberrant expression of EMT markers and poor prognosis in non-small cell lung cancer. J Cancer. 2017;8(8):1484-1491.

15. Xu GF, Zhang WJ, Sun Q, Xu X, Zou X, Guan W. Combined epithelialmesenchymal transition with cancer stem cell-like marker as predictors of recurrence after radical resection for gastric cancer. World J Surg Oncol. 2014;12:368

16. Masuda GO, Yashiro M, Kitayama K, et al. Clinicopathological correlations of autophagy-related proteins LC3, Beclin 1 and p62 in gastric cancer. Anticancer Res. 2016;36(1):129-136.

17. Gugnoni M, Sancisi V, Manzotti G, Gandolfi G, Ciarrocchi A. Autophagy and epithelial-mesenchymal transition: an intricate interplay in cancer. Cell Death Dis. 2016;7(12):e2520.

18. Yang Z, Wang H, Xia L, et al. Overexpression of PAK1 correlates with aberrant expression of EMT markers and poor prognosis in non-small cell lung cancer. J Cancer. 2017;8(8):1484-1491.

19. Szász AM, Lánczky A, Nagy Á, et al. Cross-validation of survival associated biomarkers in gastric cancer using transcriptomic data of 1,065 patients. Oncotarget. 2016;7(31):49322-49333.

20. Liu ZY, Wu T, Li Q, et al. Notch signaling components: diverging prognostic indicators in lung adenocarcinoma. Medicine. 2016;95(20):e3715.
21. D'Errico M, de Rinaldis E, Blasi MF, et al. Genome-wide expression profile of sporadic gastric cancers with microsatellite instability. Eur J Cancer. 2009;45(3):461-469.

22. Chen X, Leung SY, Yuen ST, et al. Variation in gene expression patterns in human gastric cancers. Mol Biol Cell. 2003;14(8):3208-3215.

23. Cho JY, Lim JY, Cheong JH, et al. Gene expression signature-based prognostic risk score in gastric cancer. Clin Cancer Res. 2011;17(7): 1850-1857.

24. Cao QH, Liu F, Yang ZL, et al. Prognostic value of autophagy related proteins ULK1, Beclin 1, ATG3, ATG5, ATG7, ATG9, ATG10, ATG12, LC3B and p62/SQSTM1 in gastric cancer. Am J Trans Res. 2016;8(9): 3831-3847.

25. Yoshioka A, Miyata H, Doki Y, et al. LC3, an autophagosome marker, is highly expressed in gastrointestinal cancers. Int J Oncol. 2008;33(3):461-468.

26. Su Z, Yang Z, Xu Y, Chen Y, Yu Q. Apoptosis, autophagy, necroptosis, and cancer metastasis. Mol Cancer. 2015;14:48.

27. Ding YP, Yang XD, Wu Y, Xing CG. Autophagy promotes the survival and development of tumors by participating in the formation of vasculogenic mimicry. Oncol Rep. 2014;31(5):2321-2327.

28. Yang X, Yu DD, Yan F, et al. The role of autophagy induced by tumor microenvironment in different cells and stages of cancer. Cell Biosci. 2015;5:14.

29. Zhao H, Yang M, Zhao J, Wang J, Zhang Y, Zhang Q. High expression of LC3B is associated with progression and poor outcome in triplenegative breast cancer. Med Oncol. 2013;30(1):475.

30. Tang JY, Hsi E, Huang YC, Hsu NC, Chu PY, Chai CY. High LC3 expression correlates with poor survival in patients with oral squamous cell carcinoma. Hum Pathol. 2013;44(11):2558-2562.

31. Brabletz T, Kalluri R, Nieto MA, Weinberg RA. EMT in cancer. Nat Rev Cancer. 2018;18(2):128-134.

32. Huang L, Wu RL, Xu AM. Epithelial-mesenchymal transition in gastric cancer. Am J Transl Res. 2015;7(11):2141-2158.

33. Kim MA, Lee HS, Lee HE, Kim JH, Yang HK, Kim WH. Prognostic importance of epithelial-mesenchymal transition-related protein expression in gastric carcinoma. Histopathology. 2009;54(4):442-451.

34. Gugnoni M, Sancisi V, Manzotti G, Gandolfi G, Ciarrocchi A. Autophagy and epithelial-mesenchymal transition: an intricate interplay in cancer. Cell Death Dis. 2016;7(12):e2520.

35. Qiang L, He YY. Autophagy deficiency stabilizes TWIST1 to promote epithelial-mesenchymal transition. Autophagy. 2014;10(10):1864-1865.

36. Zhao Z, Zhao J, Xue J, Zhao X, Liu P. Autophagy inhibition promotes epithelial-mesenchymal transition through ROS/HO-1 pathway in ovarian cancer cells. Am J Cancer Res. 2016;6(10):2162-2177.
Cancer Management and Research

\section{Publish your work in this journal}

Cancer Management and Research is an international, peer-reviewed open access journal focusing on cancer research and the optimal use of preventative and integrated treatment interventions to achieve improved outcomes, enhanced survival and quality of life for the cancer patient. The manuscript management system is completely online and includes

\section{Dovepress}

a very quick and fair peer-review system, which is all easy to use. Visit http://www.dovepress.com/testimonials.php to read real quotes from published authors. 